Research Article

\title{
Application of Fault Diagnosis of Seawater Hydraulic Pump Based on Transfer Learning
}

\author{
Yang Miao $\mathbb{D},{ }^{1,2}$ Yuncheng Jiang, ${ }^{1}$ Jinfeng Huang, ${ }^{1}$ Xiaojun Zhang, ${ }^{1}$ and Lei Han ${ }^{3}$ \\ ${ }^{1}$ Faculty of Materials and Manufacturing, Beijing University of Technology, Beijing, China \\ ${ }^{2}$ Beijing Key Laboratory of Advanced Manufacturing Technology, Beijing University of Technology, Beijing, China \\ ${ }^{3}$ Beijing University of Posts and Telecommunications, Beijing, China
}

Correspondence should be addressed to Yang Miao; miaoyang@vip.126.com

Received 18 October 2019; Revised 10 November 2020; Accepted 27 November 2020; Published 10 December 2020

Academic Editor: Francisco Beltran-Carbajal

Copyright ( $\odot 2020$ Yang Miao et al. This is an open access article distributed under the Creative Commons Attribution License, which permits unrestricted use, distribution, and reproduction in any medium, provided the original work is properly cited.

\begin{abstract}
The working environment of seawater axial piston hydraulic pump is harsh, and it is difficult to diagnose due to insufficient fault database. In contrast, pumps of the same type but using hydraulic oil have an adequate fault database and are easy to diagnose. In view of the above situation, a fault diagnosis method of seawater hydraulic piston pump based on transfer learning is proposed. The method decomposes the original sampled fault signal by complementary ensemble empirical mode decomposition (CEEMD) to obtain the intrinsic mode function (IMF) that can characterize the original signal. The singular value decomposition (SVD) is performed on the IMF. Then, the obtained singular value is used as a feature parameter to construct a feature vector. The feature data of seawater hydraulic pump and oil pump are used as target data and auxiliary data to form training data. The training data is trained based on the iterative adjustment of the weight through the TrAdaBoost transfer learning algorithm. Finally, the results of diagnosis and classification are compared with traditional machine learning. When the number of training data is 5 groups, the accuracy of transfer learning is $30.5 \%$ higher than that of traditional machine learning. The results show that transfer learning has great advantages in the case of a small number of samples.
\end{abstract}

\section{Introduction}

The seawater axial piston hydraulic pump is an important component of the deep-sea application. The normal operation of the seawater axial piston hydraulic pump is the core component of the hydraulic system for deep sea applications. Seawater hydraulic system requires a healthy operation of the seawater hydraulic pump. Zhai et al. [1] simulated the cavitation flow of the seawater hydraulic axial piston pump based on the Schnerr-Sauer model, and analyzed the fluctuations of the pump outlet flow and pressure under different inlet pressure conditions. Dong et al. [2] studied corrosion and wear in seawater hydraulic pumps and introduced a new type of seawater hydraulic piston pump with better suction characteristics. Nie [3] analyzed and summarized the current status of seawater hydraulic piston pumps [4]. The research progress of port valve distribution and port plate distribution is analyzed. Alobaidi [5] analyzed various levels of cavitation in different operation conditions. A detailed analysis of the results obtained from the acoustic signal was carried out to predict cavitation in the pump under different operating conditions. Alobaidi $[6,7]$ also used vibration to detect, diagnosed the cavitation phenomenon in centrifugal pumps, and analyzed the vibration signal results obtained in the time and frequency domains in order to better understand the detection of cavitation phenomenon in the pumps.

Hydraulic pump is a mechanical system, and its diagnosis is usually monitored by measuring vibration or noise signals. Empirical mode decomposition (EMD) is a signal decomposition method proposed by Huang et al. [8], which can adaptively decompose the signal to obtain a series of high-to-low intrinsic mode function (IMF). This method is widely used in signal processing. Wu et al. [9] proposed the ensemble empirical mode decomposition (EEMD) by using the statistical properties of Gaussian white noise uniform 
distribution. However, this method cannot completely neutralize the artificially added white noise, which will lead to misjudgment of fault diagnosis. The complementary ensemble empirical mode decomposition (CEEMD) reduces the residual white noise in the EEMD reconstructed signal by adding positive and negative pairs of Gaussian white noise to the original signal $[10,11]$.

Machine learning is a hot topic in today's scientific research, and it is also widely used in the field of fault diagnosis. Kankar [12] used Artificial Neural Network (ANN) and Support Vector Machine (SVM) to diagnose ball bearings [13-16] and conducted comparative experiments on the effectiveness of ANN and SVM. Shi et al. [17] proposed an early fault diagnosis method for manufacturing systems based on machine learning. The method of inductive learning is adopted to automatically obtain the statistical boundary vectors of the signal, and then a normal feature space is established, according to which an abnormal signal can be detected so that the faults in the complex system can be easily found. Alobaidi [18] extracted features from vibration signals that are used as inputs to the neural network. The method provided an intelligent system to be used in condition monitoring of centrifugal pumps. Huang et al. [19] proposed a new method for fault diagnosis hydraulic servo valve based on genetic algorithm for backpropagation neural network. Compared with other artificial neural networks, this method shortens the training time and improves the accuracy. However, the traditional machine learning method requires that the distribution of training data should be identical and sufficient [20], but seawater hydraulic pump is rarely used in engineering, and the fault data is relatively insufficient. Using seawater hydraulic pump fault data as training data is no longer suitable for traditional machine learning methods. Transfer learning is not limited to learning methods in which training data and test data have the same feature distribution. Transfer learning can learn to use the laws of knowledge in existing data to solve different but related fields, thereby effectively solving the problem of traditional machine learning.

In this paper, a fault diagnosis method for seawater axial piston hydraulic pump based on transfer learning is proposed. Section 1 introduces the signal processing method. Section 2 describes the research of transfer learning and transfer learning algorithm. Section 3 introduces the entire diagnosis process. Section 4 is to test and diagnose the fault, and diagnosis results are compared with the traditional machine learning method. This paper focuses on the application of transfer learning in fault diagnosis and proves that the transfer learning has higher diagnosis accuracy than the traditional machine learning algorithm in the case of few samples.

\section{Theory of Algorithm}

As shown in Figure 1, the complementary ensemble empirical mode decomposition (CEEMD) is used to decompose and select the original fault signal, and IMF representing the original signal is obtained. Singular value decomposition (SVD) is applied to IMF. Then, the singular value obtained by decomposition is used as the characteristic parameter. The characteristic data of seawater hydraulic pump and oil pump are used as target data and auxiliary data for training. The training data is trained by TrAdaBoost transfer learning algorithm based on iterative adjustment of weights.

\subsection{Complementary Ensemble Empirical Mode Decomposition (CEEMD)}

2.1.1. Basic Principles of CEEMD. The basic principle of CEEMD is essentially an improved algorithm based on EEMD. The decomposition process has three main steps [21-24]:

(a) Add a pair of random Gaussian white noises $n(t)$ in the signal $x(t)$ :

$$
\left\{\begin{array}{l}
x_{1}(t)=x(t)+n(t) \\
x_{2}(t)=x(t)-n(t)
\end{array}\right.
$$

(b) The EMD algorithm is used to decompose and $x_{1}(t)$ $x_{2}(t)$ to obtain IMF1 and IMF2, and the average value of each group of $I M F$ is calculated. The result is as follows:

$$
\mathrm{IMF}=\frac{\mathrm{IMF} 1+\mathrm{IMF} 2}{2} .
$$

(c) Each IMF component calculated in step (b) is recorded as $c_{i}(t)$. The signal $x(t)$ is decomposed using the CEEMD and the result is as follows:

$$
x(t)=\sum_{i=1}^{n} c_{i}(t)+r_{n}(t)
$$

where $r_{n}(t)$ is the residual.

2.1.2. Analysis of Correlation. Due to the influence of background noise and the insufficiency of the algorithm, the IMF obtained by CEEMD decomposition has false components and noise components. The correlation coefficient $\rho_{x y}$ is used to select the intrinsic mode function close to the original signal. The mathematical expression is [25]

$$
\rho_{x y}=\frac{\sigma_{x y}}{\sigma_{x} \sigma_{y}},
$$

where $\sigma_{x y}$ is the covariance of $x, y, \sigma_{x}$, and $\sigma_{y}$ is the standard deviation of $x$ and $y$.

2.2. Singular Value Decomposition. Singular value decomposition (SVD) is widely used in signal processing and fault diagnosis [26-28]. The singular value obtained by decomposition has stability invariance and can describe the intrinsic characteristics of the original signal. Orthogonal transformation is the essence of singular value decomposition (SVD). SVD is the generalization of spectrum analysis theory in the arbitrary matrix. The singular value of matrix is the inherent feature of matrix. It has very good stability. This 


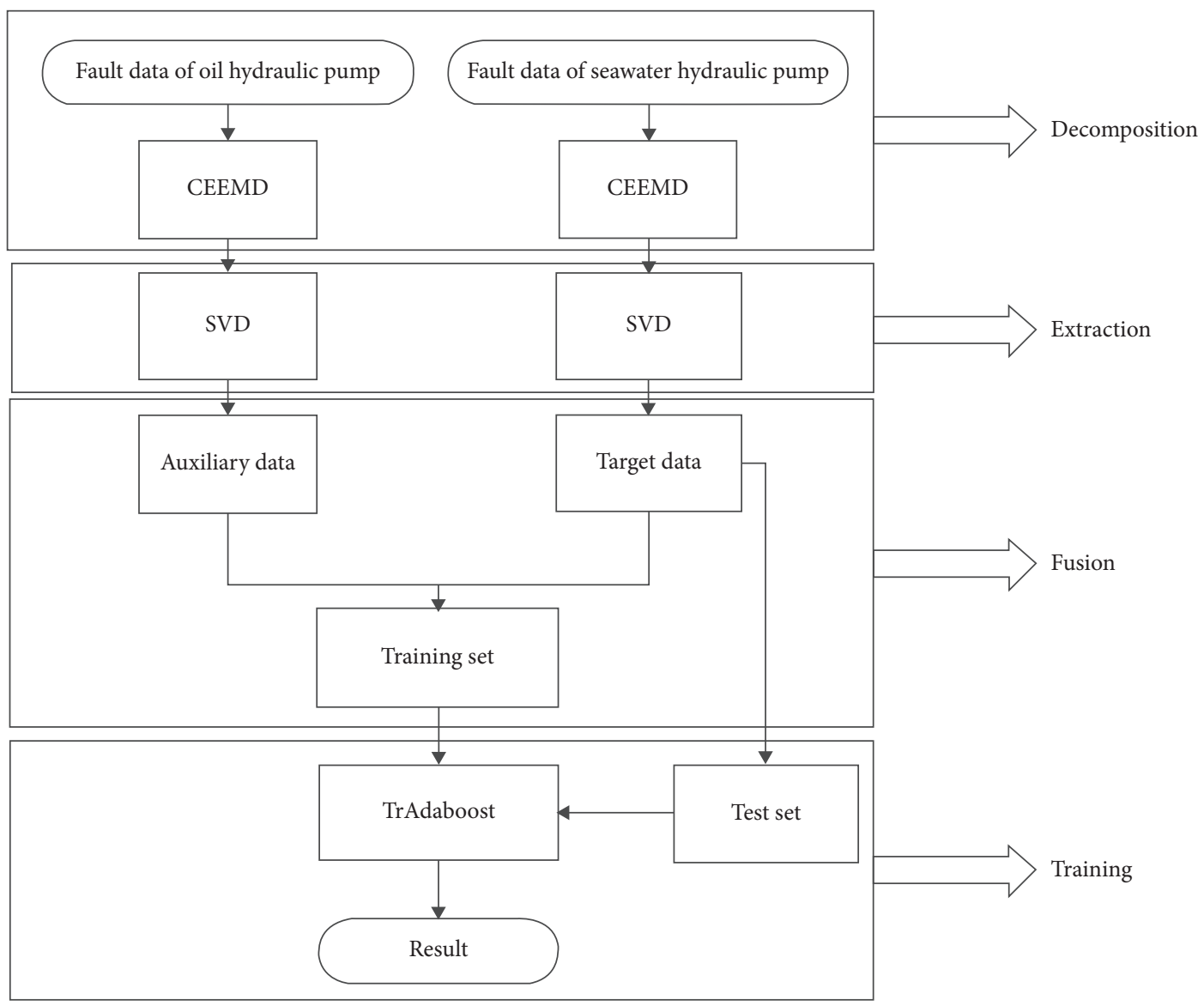

Figure 1: Method flowchart.

way, the characteristic information of the hydraulic pump failure can be effectively extracted. The specific process of singular value decomposition is as follows. is

For the matrix $A$, the singular value decomposition form

$$
A=M D N^{T},
$$

where $M$ and $N$ are standard orthogonal matrices and $D$ is a diagonal matrix:

$$
\begin{gathered}
{\left[\begin{array}{cc}
D_{1} & 0 \\
0 & 0
\end{array}\right],} \\
D_{1}=\operatorname{diag}\left(\sigma_{1}, \sigma_{2}, \ldots, \sigma_{\mathrm{r}}\right), \\
\sigma_{1} \geq \sigma_{2} \geq \ldots \sigma_{r} \geq 0,
\end{gathered}
$$

where $r$ is the rank of $A$, and the singular value vector is

$$
\left(\sigma_{1}, \sigma_{2}, \ldots, \sigma_{r}\right) \text {. }
$$

\subsection{Transfer Learning}

2.3.1. The Theoretical Concept of Transfer Learning. The phenomenon of transfer learning exists in various fields of real life. Many simple examples of daily life can also explain the rationale of transfer. For example, people who learn to ride bicycles can easily learn to ride motorcycles. People who learn English are more likely to learn Spanish. The basic idea of transfer learning is to learn the basic knowledge within a known source domain (auxiliary domain) and apply the learned knowledge to different but related unknown domains (target domains) to solve similar problems [29-32].

As shown in the following figures, different learning methods between the traditional machine learning method and the transfer learning method are, respectively, indicated. For a number of different learning tasks, the traditional machine learning method is to learn each task individually, and there is no correlation between the learning task processes; however, in the transfer learning process, a certain algorithm is used to learn knowledge from the relevant source fields and then solve the tasks or problems that exist in the target area. The difference between the two different machine learning methods can be clearly seen from Figure 2 .

2.3.2. Transfer Learning TrAdaBoost Algorithm. The TrAdaBoost transfer learning algorithm [33] is a machine learning algorithm based on iterative adjustment of weights. The learning process of the transfer learning algorithm TrAdaBoost is as follows.

The auxiliary data and the target data constitute a training sample. First, the data weight vector in the training 


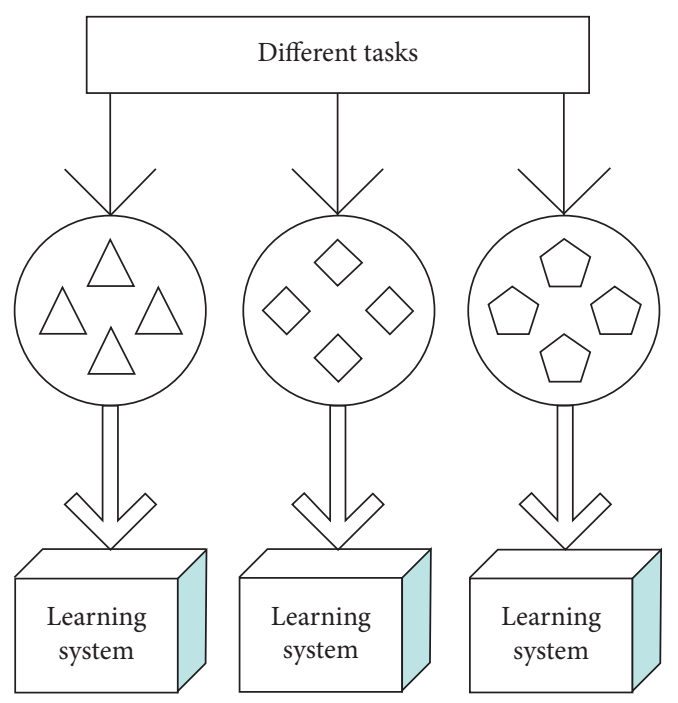

(a)

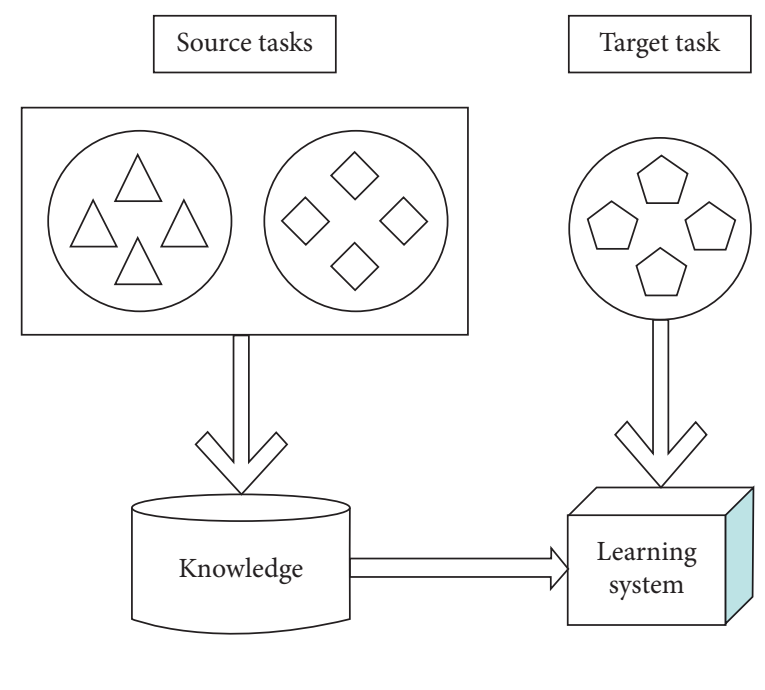

(b)

FIGURE 2: Different learning processes between (a) traditional machine learning and (b) transfer learning.

sample is initialized (the first generation weight vector is the set):

$$
\begin{aligned}
& w^{1}=\left(\omega_{1}^{1}, \ldots \omega_{n+m}^{1}\right), \\
& \omega_{i}^{1}= \begin{cases}\frac{1}{n}, & i=1, \ldots, n, \\
\frac{1}{m}, & i=n+1, \ldots n+m,\end{cases}
\end{aligned}
$$

where $n$ is the number of auxiliary data and $m$ is the number of target data.

Set the weight distribution $p^{t}$ to satisfy

$$
p^{t}=\frac{w^{t}}{\sum_{i=1}^{n+m} \omega_{i}^{t}}
$$

where $t$ is algebra, $t=1,2 \ldots N$

Based on the combined training dataset and its weight distribution and test dataset, a classifier on the test dataset is obtained $h_{t}$ and the error rate on the target data is calculated:

$$
e_{t}=\sum_{i=n+1}^{n+m} \frac{\omega_{i}^{t}\left|h_{t}\left(x_{i}\right)-c\left(x_{i}\right)\right|}{\sum_{i=n+1}^{n+m} \omega_{i}^{t}}
$$

where $c(x)$ is the label of the sample data.

Set parameters:

$$
\begin{aligned}
& \beta_{t}=\frac{e_{t}}{1-e_{t}}, \\
& \beta=\frac{1}{1+\sqrt{2 \ln n / N}} .
\end{aligned}
$$

The weight vector of the next generation:

$$
\omega_{i}^{t+1}= \begin{cases}\omega_{i}^{t} \beta^{\left|h_{t}\left(x_{i}\right)-c\left(x_{i}\right)\right|}, & i=1, \ldots, n, \\ \omega_{i}^{t} \beta_{t}^{-h_{t}\left(x_{i}\right)-c\left(x_{i}\right) \mid}, & i=n+1, \ldots n+m .\end{cases}
$$

The final classifier for the output is

$$
h(x)= \begin{cases}1, & \prod_{t=(N / 2)}^{N} \beta_{t}^{-h_{t}(x)} \geq \prod_{N / 2}^{N} \beta_{t}^{-(1 / 2)}, \\ 0, & \text { otherwise. }\end{cases}
$$

It can be seen that, in each iteration of the round if an auxiliary training data is misclassified, the weight of the data can be reduced. Specifically, the data is multiplied by $\beta^{\left|h_{t}\left(x_{i}\right)-c\left(x_{i}\right)\right|}$, where the value $\beta$ is between 0 and 1 . Therefore, in the next iteration, the misclassified sample will have less impact on the classification model than the previous round. After the iteration, the data in the auxiliary data that meets the target data will have a higher weight, and the weight of the auxiliary data that does not meet the target data will gradually decrease. Finally, the classification model is obtained.

\section{Experimentation}

In order to verify the effectiveness of the method proposed in this paper, the experimental platform of oil hydraulic and seawater hydraulic piston pump is built. The experimental schematic diagram is shown in Figure 3. The piston pump is driven by $45 \mathrm{KW}$ variable frequency AC motor. The rated pressure of the pump is $21 \mathrm{MPa}$ and the rated speed is $4000 \mathrm{r} / \mathrm{m}$. Therefore, the corresponding shaft frequency is 6.7 Hz. In order to monitor the comprehensive health status of the pump, vibration sensor, pressure sensor, and flow 


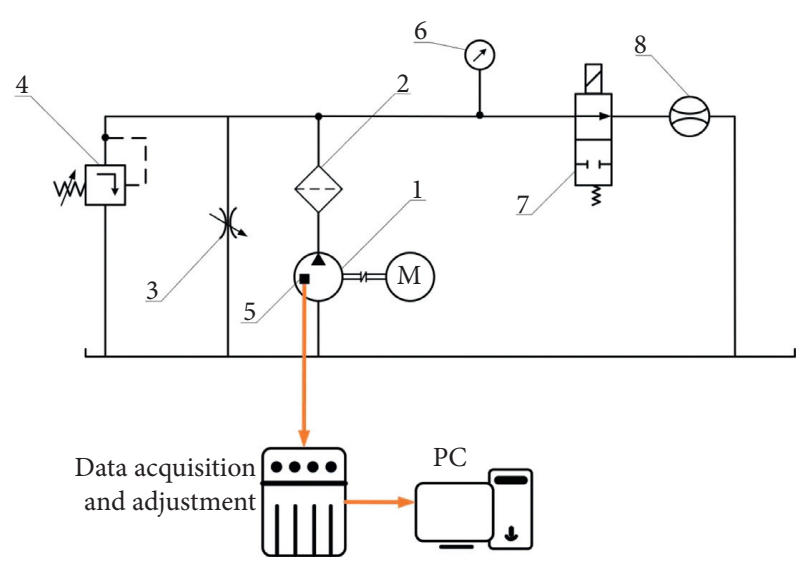

FIgURE 3: Experimental schematic diagram. (1) Seawater axial piston hydraulic pump. (2) Filter. (3) Throttle valve. (4) Safety valve. (5) Vibration sensor. (6) Pressure sensor. (7) Switch valve. (8) Flow sensor.

sensor are installed on the piston pump assembly. The model of vibration sensor is ULT2059, the measuring range is $500 \mathrm{~g}$, and the accuracy is $50 \mathrm{mV} / \mathrm{g}$. The pressure sensor model is MPM480, and the range is $(0-30) /(0-1) \mathrm{MPa}$. The model of flow sensor is CLG-15, and the range is $(0-5) /(4.2-120) \mathrm{L} / \mathrm{min}$. All sensors have $24 \mathrm{~V}$ DC power supply.

The vibration sensor is used to measure the radial vibration signal of the piston pump. The measurement error of acceleration sensor is $2.5 \mathrm{~g}$. The radial vibration sensor is installed near the swash plate trunnion of the plunger pump, and the sampling frequency of the sensor is $2 \mathrm{KHz}$. The pressure sensor is used to measure the pressure signal at the inlet and outlet of the piston pump. LabWindows/CVI + RTX is used to edit the interactive interface of the host computer, and the latter is used to realize the communication between the software and the sensor and complete the analog to digital (A/D) conversion of the sensor input signal.

3.1. Uncertainty of Acceleration. The relative expanded uncertainty of acceleration sensor is $u_{\text {rel }}(A)=1.0 \%$. The relative standard uncertainty is

$$
u_{\text {rel }}(A)=\frac{U_{\text {rel }}(A)}{2}=0.5 \% \text {. }
$$

The relative expanded uncertainty of data acquisition system is $u_{\text {rel }}(I)=0.1 \%$. The relative standard uncertainty is

$$
u_{\text {rel }}(I)=\frac{U_{\text {rel }}(I)}{2}=0.05 \% \text {. }
$$

Since the above standard uncertainty components are independent of each other, the combined standard uncertainty is as follows:

$$
u_{\text {crel }}(A)=\sqrt{u^{2_{\text {rel }}}(A)+u^{2_{\text {rel }}}(I)}=0.502 \% .
$$

\section{Result and Discussion}

Taking a group of bearing fault data in the oil hydraulic pump data as an example, the CEEMD is used to decompose the sampling signal to obtain nine IMF and calculate the correlation coefficient between each IMF and the original signal. As shown in Table 1, the top 6 IMF of the 9 IMF had large correlation with the original signal, so they are selected as the data for use. As shown in Figure 4, the top 6 IMF components are selected.

The top 6 IMF components are selected, and SVD decomposition is performed on the IMF to calculate the singular values of sample data as a group of training data: $(26.698,9.803,9.203,6.541,6.222,4.893)$

All the sample data are decomposed by CEEMD and SVD to form the training dataset. The data of seawater hydraulic pump is the target data, and the data of oil hydraulic pump is used as auxiliary data. In order to compare the two learning methods, the experimental data and feature extraction methods are unchanged, and the transfer learning algorithm is compared with the traditional machine learning algorithm. The auxiliary data is set to 100 groups. When the two learning modes have different sets of target data, the final fault diagnosis accuracy rate is compared.

It can be seen from Figure 5 that, in the case of a small amount of target data, the accuracy of conventional machine learning cannot be performed or the fault diagnosis is very low, and the transfer learning has a higher accuracy rate in this case. The smaller the number of target data, the more obvious the advantages of transfer learning. When the number of target data is 5 groups, the accuracy of transfer learning is $30.5 \%$ higher than that of SVM. The reason is that the classifiers trained by traditional machine learning are not accurate. However, transfer learning not only uses the target number data for training but also uses the data in the auxiliary data similar to the target data for training, which can train a better classifier.

As the number of target data increases, the diagnostic accuracy rate of transfer learning and traditional machine learning will also increase. The accuracy of traditional machine learning and transfer learning is the same when the number of target data increases to 25 groups. When the number of target data continues to increase, the diagnostic accuracy of traditional machine learning is slightly higher than that of transfer learning. When the amount of target data is large enough, both learning methods can achieve good performance. The reason for the above phenomenon is that when the number of target data increases, the amount of training data of the conventional machine learning increases and the accuracy of the trained classifier increases. With the increase of the amount of target data of transfer learning, the TrAdaBoost algorithm can filter out more auxiliary data similar to the target data to train a higher precision classifier. When the amount of target data increases to a certain amount, the traditional machine 
TABLE 1: Correlation coefficient between IMF and signal.

\begin{tabular}{|c|c|c|c|c|c|c|c|c|c|}
\hline IMF & IMF1 & IMF2 & IMF3 & IMF4 & IMF5 & IMF6 & IMF7 & IMF8 & IMF9 \\
\hline Correlation coefficient & 0.838 & 0.282 & 0.277 & 0.180 & 0.171 & 0.112 & 0.083 & 0.008 & 0.003 \\
\hline
\end{tabular}
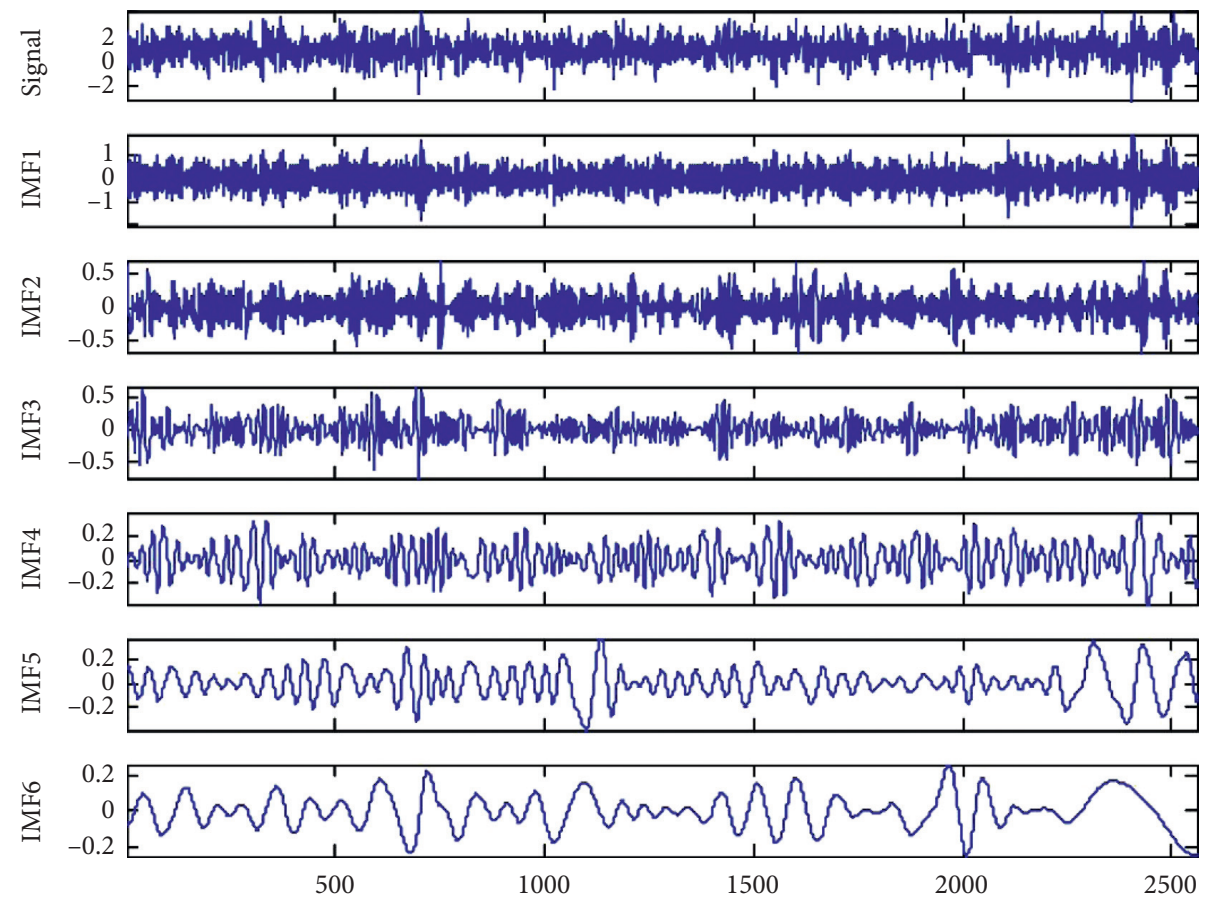

Figure 4: CEEMD results of signal.

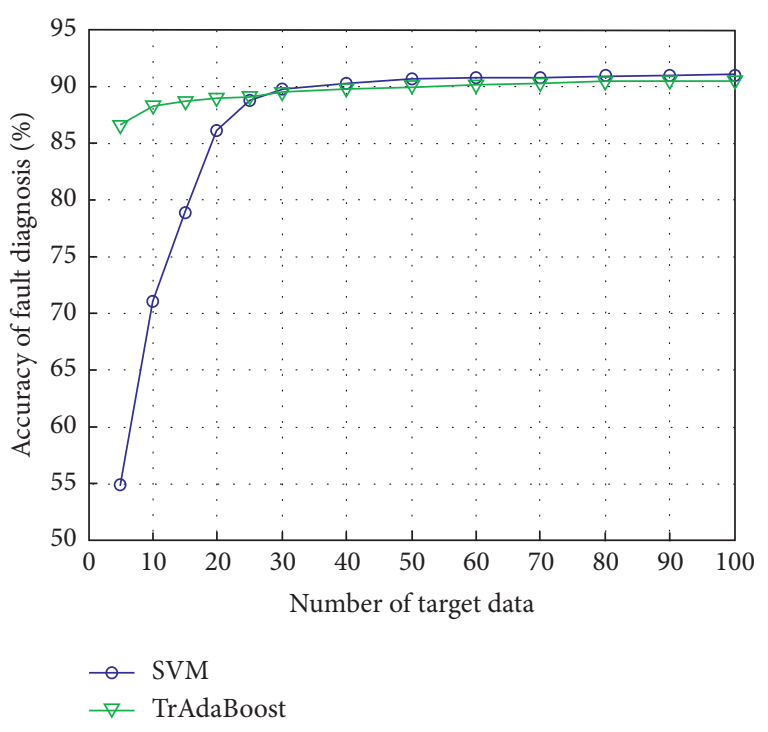

FIGURE 5: Comparison of correct rate of transfer learning and machine learning diagnosis.

learning has enough training data to train high-precision classifiers. However, during the transfer learning process, the small amount of noise data included in the auxiliary data in the training set will have a little influence on the accuracy of the trained classifier. Therefore, when the target data volume is sufficient, the diagnostic accuracy rate of the traditional machine learning is better than that of the transfer learning.

\section{Conclusion}

A fault diagnosis method for seawater hydraulic pump based on transfer learning is proposed. The method uses CEEMD and SVD for data feature extraction. The feature data of seawater hydraulic pump and oil pump are used as target data and auxiliary data to form training data. In the iterative learning process of transfer learning, valuable and important sample weights are increased from generation to generation, but the weights of the secondary sample are reduced from generation to generation. Finally, compared with SVM, the results of transfer learning still have a high accuracy rate in the case of few target training data. The method solves the limitation that the traditional machine learning is not applicable under the condition that the training data and the test data do not have the same feature, and target training data is few. The fault diagnosis method based on transfer learning will also be applicable to the fault 
diagnosis of bearings or gears under variable working conditions.

\section{Data Availability}

The experimental data used to support the findings of this study were supplied by the research group of Key Laboratory of Advanced Manufacturing Technology under license and so we cannot put it into the system, and the requests for access to these data should be made to the corresponding author.

\section{Conflicts of Interest}

The authors declare no conflicts of interest.

\section{Acknowledgments}

The authors would like to appreciate the support of National Natural Science Foundation of China (51605009, 51975011, and 51705026) and Fundamental Research Funds for the Central Universities (2019PTB-007).

\section{References}

[1] J. Zhai, Y. Zhao, and H. Zhou, "Numerical simulation of cavitating flow in seawater hydraulic axial piston pump," Nongye Jixie Xuebao/Transactions of the Chinese Society of Agricultural Machinery, vol. 43, no. 11, pp. 244-249, 2012.

[2] W. Dong, L. Zhuangyun, and Z. Yuquan, "Study of the key problems in a seawater hydraulic piston pump and its applications," Industrial Lubrication \& Tribology, vol. 53, no. 5, pp. 211-216, 2001.

[3] S. L. Nie, "Progress and prospect of seawater hydraulic piston pump," Chinese Hydraulics \& Pneumatics, vol. 1, 2015.

[4] A. Halanay, C. A. Safta, I. Ursu, and F. Ursu, "Stability of equilibria in a four-dimensional nonlinear model of a hydraulic servomechanism," Journal of Engineering Mathematics, vol. 49, no. 4, pp. 391-405, 2004.

[5] A. R. Alobaidi, "Experimental investigation of the effect of suction valve opening on the performance and detection of cavitation in the centrifugal pump based on acoustic analysis technique," Archives of Acoustics, vol. 44, no. 1, pp. 59-69, 2019.

[6] A. R. Alobaidi, "Investigation of effect of pump rotational speed on performance and detection of cavitation within a centrifugal pump using vibration analysis," Heliyon, vol. 5, no. 6, Article ID e01910, 2019.

[7] A. R. Alobaidi, "Detection of cavitation phenomenon within a centrifugal pump based on vibration analysis technique in both time and frequency domains," Experimental Techniques, vol. 44, no. 2, prepublish, 2020.

[8] E. Huang Norden, S. Zheng, R. Long Steven et al., "The empirical mode decomposition and the Hilbert spectrum for nonlinear and non-stationary time series analysis," Proceedings of the Royal Society A: Mathematical, Physical and Engineering Sciences, vol. 454, no. 1971, 1998.

[9] Z. Wu, N. E. Huang, and X. Chen, "The multi-dimensional ensemble empirical mode decomposition method," Advances in Adaptive Data Analysis, vol. 1, no. 3, pp. 339-372, 2009.

[10] J.-R. Yeh, J.-S. Shieh, and N. E. Huang, "Complementary ensemble empirical mode decomposition: a novel noise enhanced data analysis method," Advances in Adaptive Data Analysis, vol. 2, no. 2, pp. 135-156, 2010.

[11] Z. Liye, Y. Wei, and Y. Ruqiang, "Gearbox fault diagnosis using complementary ensemble empirical mode decomposition and permutation entropy," Shock and Vibration, vol. 2016, Article ID 3891429, 8 pages, 2016.

[12] P. K. Kankar, S. C. Sharma, and S. P. Harsha, "Fault diagnosis of ball bearings using machine learning methods," Expert Systems with Applications, vol. 38, no. 3, pp. 1876-1886, 2011.

[13] F. Zhang, J. Huang, F. Chu, and L. Cui, "Mechanism and method for the full-scale quantitative diagnosis of ball bearings with an inner race fault," Journal of Sound and Vibration, vol. 488, no. 8, Article ID 115641, 2020.

[14] F. Zhang, J. Huang, F. Chu, and L. Cui, "Mechanism and method for outer raceway defect localization of ball bearings," IEEE Access, vol. 8, pp. 4351-4360, 2020.

[15] L. Cui, Z. Jin, J. Huang, and H. Wang, "Fault severity classification and size estimation for ball bearings based on vibration mechanism," IEEE Access, vol. 7, pp. 56107-56116, 2019.

[16] L. Cui, X. Wang, H. Wang, and J. Ma, "Research on remaining useful life prediction of rolling element bearings based on time-varying kalman filter," IEEE Transactions on Instrumentation and Measurement, vol. 69, no. 6, pp. 2858-2867, 2020.

[17] W.-Wu Shi, H.-S. Yan, and K.-P. Ma, “A new method of early fault diagnosis based on machine learning," in Proceedings of 2005 International Conference on Machine Learning and Cybernetics, Guangzhou, China, August 2005.

[18] A. R. Al-Obaidi and H. Towsyfyan, “An experimental study on vibration signatures for detecting incipient cavitation in centrifugal pumps based on envelope spectrum analysis," Journal of Applied Fluid Mechanics, vol. 12, no. 6, pp. 20572067, 2019.

[19] H. Huang, K.-S. Chen, and L.-C. Zeng, "BP neural networkbased on fault diagnosis of hydraulic servo-valves," in Proceedings of 2005 International Conference on Machine Learning and Cybernetics, Guangzhou, China, August 2005.

[20] J. Ramon, K. Driessens, and T. Croonenborghs, "Transfer learning in reinforcement learning problems through partial policy recycling," in Proceedings of the 18th European Conference on Machine Learning, September 2007.

[21] L. Tang, W. Dai, L. Yu, and S. Wang, "A novel CEEMD-based EELM ensemble learning paradigm for crude oil price forecasting," International Journal of Information Technology \& Decision Making, vol. 14, no. 1, pp. 141-169, 2015.

[22] Y. Wang, C. Lu, H. Liu et al., "Fault diagnosis for centrifugal pumps based on complementary ensemble empirical mode decomposition, sample entropy and random forest," in Proceedings of the 2016 12th World Congress on Intelligent Control and Automation (WCICA), June 2016.

[23] A.-O. Boudraa and J.-C. Cexus, "EMD-based signal filtering," IEEE Transactions on Instrumentation and Measurement, vol. 56, no. 6, pp. 2196-2202, 2007.

[24] Z. Zhi, y. S. Zhu, Y. Y. Zhang et al., "Adaptive fault diagnosis of rolling bearings based on EEMD and demodulated resonance," Journal of Vibration \& Shock, vol. 32, no. 2, pp. 76-80, 2013.

[25] F. Jiang, Z. Zhu, W. Li et al., "A fusion feature extraction method using EEMD and correlation coefficient analysis for bearing fault diagnosis," Applied Sciences, vol. 8, no. 9, 2018.

[26] H. Zhao, H. Shen, Y. Fu et al., "Using singular value decomposition and high order spectrum for bearings fault diagnosis," in Proceedings of the 2014 IEEE Conference and Expo 
Transportation Electrification Asia-Pacific (ITEC Asia-Pacific), August 2014.

[27] H. Xu, Y. Fan, J. Wu et al., "Bearing fault diagnosis method based on singular value decomposition and hidden markov model," in Proceedings of the 27th Chinese Control and Decision Conference (2015 CCDC), May 2015.

[28] J. Cheng, D. Yu, J. Tang, and Y. Yang, "Application of SVM and SVD technique based on EMD to the fault diagnosis of the rotating machinery," Shock and Vibration, vol. 16, no. 1, pp. 89-98, 2009.

[29] S. J. Pan and Q. Yang, “A survey on transfer learning," IEEE Transactions on Knowledge and Data Engineering, vol. 22, no. 10, pp. 1345-1359, 2010.

[30] A. Arnold, R. Nallapati, and W. W. Cohen, "A comparative study of methods for transductive transfer learning," in Proceedings of the Seventh IEEE International Conference on Data Mining Workshops (ICDMW 2007), March 2008.

[31] R. Zhang, H. Tao, L. Wu et al., "Transfer learning with neural networks for bearing fault diagnosis in changing working conditions," IEEE Access, vol. 5, pp. 14347-14357, 2017.

[32] L. Wen, L. Gao, and X. Li, "A new deep transfer learning based on sparse auto-encoder for fault diagnosis," IEEE Transactions on Systems, Man, and Cybernetics: Systems, vol. 49, no. 1, pp. 136-144, 2017.

[33] W. Dai, Q. Yang, G. Rong Xue, and Y. Yu, "Boosting for transfer learning," in Proceedings of the 24th International Conference on Machine Learning (ICML-2007), Corvallis, USA, June 2007. 